koagulation zwingend benötigen. Die sogenannte Tripletherapie unter Verwendung von Marcumar und einer dualen antithrombozytären Medikation wird in den aktuellen Leitlinien empfohlen, auch wenn das absolute Blutungsrisiko im ersten Jahr bis zu 20\% betragen kann, im Gegensatz zu 3\% in der APPRAISE-2-Studie.
Neue Antikoagulanzien wie Apixaban können in diesem spezifischen klinischen Kontext das Blutungsrisiko gegenüber Marcumar unter Umständen signifikant senken. Die APPRAISE-II-Studie kann damit sehr gut den Rahmen für die Planung zukünftiger Studien zur Sicherheit der oftmals notwendigen Tripletherapie vorgeben.
Prof. Dr. med. Peter W. Radke,

Prof. Dr. med. Heribert Schunkert Universitätsklinikum Schleswig-Holstein, Campus Lübeck II

II Alexander JH et al. Apixaban with Antiplatelet Therapy after Acute Coronary Syndrome. N Engl J Med 2011;365:699-708

\title{
Kritische Extremitätenischämie
}

\section{Angiogenese-Gentherapie schützt nicht vor Amputation}

\begin{abstract}
Die Behandlung von Patienten mit einer kritischen Extremitätenischämie ist nach wie vor eine Herausforderung. Auf die Angiogenese-Gentherapie mit Wachstumsfaktoren wie dem rekombinanten DNA-Plasmid, das für den humanen Fibroblastenwachstumsfaktor (FGF-1) kodiert, wurden in den letzten Jahren große Hoffnungen gesetzt. Doch in der TAMARIS-Studie blieb das Verfahren erfolglos.
\end{abstract}

FGF-1 induziert und/oder fördert die Angiogenese über eine Stimulation von Zellwachstum und -migration. Nicht virales FGF1 (NV1FGF) wird intramuskulär in die Wadenund Oberschenkelmuskulatur gespritzt und führt zur Expression von humanem FGF-1Protein. Das Risiko für systemische Nebenwirkungen sollte sehr gering sein. Behandlungsziel soll die Wiederherstellung eines funktionsfähigen Gefäßnetzes in der Nähe der Applikationsstelle sein.

In einer sehr aufwändigen randomisierten, doppelblinden, placebokontrollierten Studie wurde die Verträglichkeit und Wirksamkeit dieser Therapie bei Patienten mit kritischer Extremitätenischämie und Hautläsionen untersucht. Bei allen Patienten musste belegt sein, dass eine gefäßchirurgische Therapie bzw. eine Katheterintervention nicht mehr möglich war. 525 Patienten wurden in die Studie aufgenommen. Sie erhielten nach extensiven Voruntersuchungen (vor allem Ausschluss einer bösartigen Grunderkrankung) entweder NV1FGF oder Placebo alle zwei Wochen über einen Zeitraum von sechs Wochen intramuskulär verabreicht.

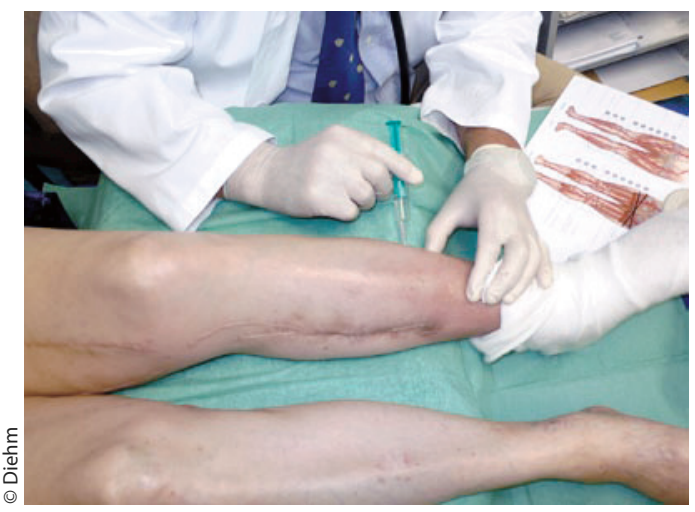

Intramuskuläre Injektion von NV1FGF bei einem Patienten mit kritischer Extremitätenischämie.

Der primäre Endpunkt der Studie war die Überlegenheit von NV1FGF gegenüber Placebo bei der Vermeidung großer Amputationen (Unterschenkel- oder Oberschenkelamputation) über einen Zeitraum von zwölf Monaten.

Nach einem Jahr konnten zwischen den beiden Behandlungsgruppen keine Unterschiede bezüglich größerer Amputationen oder Todesfällen festgestellt werden (Hazard Ratio $=1,11 ; 95 \%$-Konfidenzintervall: 0,83$1,49 ; p=0,48)$. Auch die Zeitspanne bis zur Amputation war in beiden Gruppen nicht unterschiedlich.

Wesentliche Nebenwirkungen, die bei einer Gentherapie theoretisch auftreten konnten (proliferative Retinopathie, Makulaödeme, akute ischämische Ereignisse bzw. eine Verschlechterung der Nierenfunktion) wurden nicht beobachtet.

Demnach konnte die TAMARIS-Studie keine Hinweise liefern, dass diese neuartige
Gentherapie mit NV1FGF Amputationen oder Todesfälle verhindern kann.

II Kommentar: In vorausgegangenen Tierstudien haben sich durchweg günstige Effekte einer derartigen Gentherapie in Ischämiemodellen gezeigt. Junge, gesunde Tiere sind aber wahrscheinlich mit älteren polymorbiden Gefäßpatienten schwer oder nicht vergleichbar. Die betroffenen Patienten haben meist eine generalisierte Atherothrombose mit koronarer Herzkrankheit und zerebralen Durchblutungsstörungen und zusätzlich meist ein Risikofaktorenbündel mit Bluthochdruck, Fettstoffwechselstörung und Diabetes. Es ist denkbar, dass die Gentherapie bei älteren schwer gefäßkranken Patienten nicht mehr wirksam ist.

Es ist auch denkbar, dass leitliniengerechte begleitende Medikamente wie ACEHemmer und Statine die erwünschte Angiogenese inhibieren. Außerdem könnten die optimale Dosierung und die notwendige Therapiezeit noch nicht gefunden und Vektorprobleme noch nicht gelöst sein.

Prof. Dr. med. Curt Diehm, Klinikum Karlsbad-Langensteinbach In

II Belch J et al. Effect of fibroblast growth factor NV1FGF on amputation and death: a randomised placebo-controlled trial of gene therapy in critical lim ischaemia. Lancet 2011;377:1929-1937 